\title{
Oximetría cerebral no invasiva: su uso en manejo de onfalocele gigante
}

\author{
Mena M. ${ }^{1,2}$, Selamé,R. ${ }^{1,2}$, Corral G. ${ }^{1,2}$, Córdova S. ${ }^{1,2}$, Araya C. ${ }^{1,2}$ \\ 1 Hospital Gustavo Fricke, Viña del Mar, Chile. \\ 2 Universidad de Valparaíso, Viña del Mar, Chile.
}

Introducción: Más de un 10\% de los recién nacidos en todo el mundo son prematuros. Estos pacientes tienen alto riesgo de tener secuelas tardías del neurodesarrollo por daño cerebral.

La oximetría cerebral no invasiva utilizando la tecnología NIRS (near infrared spectroscopy) es útil para detectar isquemia-hipoxia cerebral relacionada con hipoperfusión e hipoxemia y determinar distintas intervenciones para prevenir o disminuir la injuria por hipoxia cerebral.

Uno de los factores involucrados en la injuria cerebral relacionada a la perfusión en el paciente prematuro es atribuida a la falta de autorregulación cerebrovascular.

Método: Recién nacido de pre término (RNPT) de 38 semanas de edad gestacional, nacido a las 36 semanas, con antecedentes de onfalocele gigante y distrés respiratorio recuperado. Ingresa a pabellón para manejo operatorio de su onfalocele.

La monitorización estándar incluyó: saturometría de O2 (SpO2), presión arterial no invasiva (PANI), electrocardiograma, temperatura y $\mathrm{CO} 2$ espirado (etCO2).

Previo a la inducción anestésica se colocaron los sensores de NIRS en la región frontoparietal izquierda y derecha, midiéndose la SrO2C. El valor basal obtenido fue $82 \%$ izquierda/ $86 \%$ derecha. La PANI basal fue de $46 \mathrm{~mm} \mathrm{Hg}$.

Para la inducción y mantención anestésica se utilizó sevoflurano, suplementado con fentanilo y atracurio.

La ventilación mecánica controlada fue iniciada con un etCO2 basal de $49 \mathrm{~mm} \mathrm{Hg}$. Durante la manipulación del onfalocele, en el intento de introducir todo el defecto, en forma inadvertida por los cirujanos se produjo una rotación del pedículo hepático que se evidenció por una brusca caída de la SrO2C con valores de NIRS de $50 \%$ a izquierda y $57 \%$ a derecha, caída de la PAM bajo $32 \mathrm{~mm} \mathrm{Hg}$ y etCO2 a $28 \mathrm{~mm} \mathrm{Hg}$. Los cirujanos fueron advertidos de los profundos cambios hemodinámicos y de oxigenación, logrando reconocer el problema y liberar la rotación, con lo que se produce un aumento progresivo de la $\mathrm{SrO} 2 \mathrm{C}$, seguido de un incremento de la PAM y etCO2.

Resultado y Discusión: La SrO2c medida utilizando la tecnología NIRS es un indicador indirecto de flujo sanguíneo cerebral (FSC) y perfusión cerebral. El descenso de la SrO2C se correlacionó directamente con la caída de la PAM y etCO2.

El presente caso nos ilustra el potencial valor del uso de NIRS en el ajuste de los parámetros hemodinámicos y ventilatorios y en diagnosticar y sugerir al equipo quirúrgico cambios necesarios en el curso de la cirugía.

https://doi.org/10.25237/congresoclasa2019.16 